\title{
Differences in Palliative Care Delivery Among Adults With Cancer and With Terminal Noncancer Illness in Their Last Year of Life
}

Quinn KL, Wegier P, Stukel TA, et al. Comparison of palliative care delivery in the last year of life between adults with terminal noncancer illness or cancer. JAMA Netw Open. 2021;4(3):e210677. doi:10.1001/jamanetworkopen.2021.0677

\section{Study Overview}

objective. To examine the patterns in palliative care delivery in the last year of life among adults with cancer compared with adults with a noncancer terminal diagnosis.

Design. Population-based cohort study in Ontario, Canada, using linked administrative and clinical databases. The study included all adults ages 18 and over who died of cancer or noncancer terminal illnesses and received physician-delivered palliative care that was initiated in the last year of life between January 2010 and December 2017. These palliative care services are identified through the use of claims fee codes by physicians that account for delivery of palliative care, such as symptom management and counseling, that are intended to be palliative rather than curative. Exclusion criteria include patients who had 2 or more palliative care service claims the year prior to the last year of life, which may indicate existing palliative care services rather than initiation of new palliative care services in the last year of life. Other patients who were excluded from the study had palliative care services initiated within 7 days of death, as it is less likely that services and support would be arranged prior to death given the short time frame. The types of noncancer illnesses included heart failure, chronic obstructive pulmonary disease, end-stage renal disease, cirrhosis, stroke, and dementia. For the comparison of palliative care services, types of illnesses were divided into cancer, chronic organ failure (heart failure, chronic pulmonary disease, end-stage renal disease, cirrhosis, or stroke), and dementia, as they may represent different trajectories of illnesses and needs.

Setting and participants. The study included 145709 adults who died during the study period, among 351941 adults who died from illnesses described above. Another 105587 were excluded because there were no palliative care services before death, 48525 were excluded because of existing palliative care services prior to the last year of life, and 44164 were excluded because palliative care was initiated within 7 days of death. Among the study population included, 21054 died of chronic organ failure, 14033 died of dementia, and 110622 died of cancer. The median age of the study population was 78 years, with an interquartile range of 67 to 86 years, and $50.7 \%$ were female. Approximately $12.8 \%$ of the study population reside in

\section{Outcomes Research in Review Section Editors}

\begin{abstract}
KatRina F. Mateo, PhD, MPH
CUNY School of Public Health New York, NY
\end{abstract}

\author{
DANIEL ISAAC, DO, MS \\ Michigan State University \\ East Lansing, Ml
}

FRED Ko, MD, MS

Icahn School of Medicine at Mount Sinai New York, NY

\author{
TAISHI HIRAI, MD \\ University of Missouri \\ Columbia, $\mathrm{MO}$
}

WiLliam Hung, MD, MPH

Icahn School of Medicine at Mount Sinai New York, NY 
rural areas; median frailty score (hospital frailty risk score) among those who died of chronic organ failure was 10, and the score among those who died of dementia was 13. The frailty score among those who died of cancer was 3 , indicating less frailty. Those who died of organ failure and dementia also had a high mean number of prescription medications (18 and 16, respectively) compared with those with cancer (11).

Main outcome measures. Study outcome measures include the timing of palliative care initiation (primary outcome), categorized into time frames of $\leq 30$ days, 31 to 90 days, and $>90$ days before death; location of initiation of palliative care services, categorized into clinic, home, hospital, subacute care, and case management; models of care, categorized as generalist, consultative, or specialist palliative care; total number of palliative care visits before death; and location of death. The models of palliative care delivery were categorized based on the proportion of palliative care fee codes claimed by physicians. Physicians whose annual billing included more than $10 \%$ of palliative care service codes were considered palliative care specialists. Using this designation, models of palliative care were categorized into those delivered by palliative care specialists, generalists (nonpalliative care specialists), or both.

Main results. The study found that the timing of palliative care initiation was earlier among those who died of cancer compared with those with organ failure or dementia (28.9\% vs $15.9 \%$ and $15.3 \%$, respectively). After adjustment, those who died of organ failure and those who died of dementia were less likely to have palliative care services initiated $>90$ days prior to death (odds ratio [OR] 0.48 and 0.42 , respectively) and between 31 to 90 days prior to death (OR 0.77 and 0.60 , respectively), when compared with those who died of cancer (who served as the reference group). Regarding location of palliative care initiation, adults who died of cancer were less likely to have palliative care services initiated at home (14.5\%) compared with those who died of organ failure (32.8\%) or dementia (27.9\%). Overall, those who died of cancer received more palliative care visits from initiation to death (median of 11 visits) compared with those who died of organ failure (median 4 visits) and dementia (median 4 visits).
Regarding models of palliative care delivery, a higher proportion of palliative care was delivered by palliative care specialists rather than generalists among cancer patients (72.9\%) compared with those with organ failure (43.3\%) or dementia (40.1\%). The proportion of patients with cancer who died at home was $62.6 \%$, which was higher than those with organ failure (53.3\%) but lower than those with dementia (75\%).

Conclusion. There are differences in the delivery of palliative care among patients with cancer and other noncancer terminal illnesses, including timing of initiation of palliative care services, location of services, number of visits, and delivery by types of practitioners of palliative care. Understanding these disparities and targeting them are potentially important steps to ensuring appropriate access to palliative care across settings and disease types.

\section{Commentary}

Palliative care improves the quality of life of patients with serious illnesses and reduces symptom burden, and results in better satisfaction and less burdensome care. ${ }^{1}$ Although palliative care approaches have been championed for cancer management, there is increasing evidence that palliative care also improves outcomes for patients with noncancer illnesses such as heart failure. ${ }^{2}$ This study highlights the differences in palliative care delivery for patients who have cancer and noncancer diagnoses, demonstrating that timing, location, and care delivery models differ among patients with different diagnoses. The finding that noncancer terminal illness often has later palliative care initiation is a significant one, as early palliative care has been associated with improved patient outcomes; ${ }^{3}$ thus, efforts to initiate palliative care earlier in the course of illness may benefit these patients.

A particular challenge in determining when to initiate palliative care lies in predicting outcomes, ${ }^{4}$ particularly for different types of illnesses, which may have different trajectories of advancing disease and functional change. Recent research has tested novel prognostic approaches, such as using machine learning to generate mortality estimates and integrating them into clinical decision support. ${ }^{5}$ These approaches may have the potential 
to enhance palliative care delivery and may be adapted to be used in managing patients with noncancer illnesses as well. The study also found that patients with cancer were more likely to receive palliative care from specialists rather than generalists, although this could be due to how palliative care is integrated in hospitals, clinics, and systems of care that serve patients with cancer. Identifying approaches that yield better palliative care models and delivery may help to further enhance care for patients with noncancer illnesses.

\section{Applications for Clinical Practice}

Identifying differences in patterns of palliative care delivery among those with cancer and other diagnoses may be an important step towards identifying gaps and avenues to improve palliative care delivery. The underlying reasons for these differences could be targeted so that patients across settings and diagnoses may have equal access to palliative care to improve their symptoms and quality of life. Policy makers and health system leaders may consider learning from how palliative care has been integrated into oncology care, to help transform care delivery for other noncancer terminal illnesses. It may also involve broadening education to providers in different specialties, so that the value and importance of palliative care may be recognized beyond oncological care.

-William W. Hung, MD, MPH doi: $10.12788 /$ jcom.0050

\section{References}

1. Kavalieratos D, Corbelli J, Zhang D, et al. Association Between Palliative Care and Patient and Caregiver Outcomes: A Systematic Review and Meta-analysis. JAMA. 2016;316(20):2104-2114.

2. Quinn KL, Stukel T, Stall NM, et al. Association between palliative care and healthcare outcomes among adults with terminal non-cancer illness: population based matched cohort study. BMJ. 2020;370:m2257.

3. Temel JS, Greer JA, Muzikansky A, et al. Early palliative care for patients with metastatic non-small-cell lung cancer. $N$ Engl J Med. 2010;363:733-742.

4. White N, Reid F, Harris A, et al. A Systematic Review of Predictions of Survival in Palliative Care: How Accurate Are Clinicians and Who Are the Experts? PLoS One. 2016;11(8):e0161407.

5. Manz CR, Parikh RB, Small DS, et al. Effect of Integrating Machine Learning Mortality Estimates With Behavioral Nudges to Clinicians on Serious Illness Conversations Among Patients With Cancer: A Stepped-Wedge Cluster Randomized Clinical Trial. JAMA Oncol. 2020;6(12):e204759.

\section{CALL for REVIEWERS}

The Journal of Clinical Outcomes Management welcomes applications

from physicians, directors of pharmacy, nurse leaders,

and others working in ambulatory, inpatient, and long-term care

to serve as peer reviewers.

You will not be asked to review more than 3 times per year.

The following medical specialties are especially of interest: cardiology, critical care, endocrinology, hematology/oncology, hospital medicine, infectious diseases, mental health, neurology, nephrology, pulmonary disease, and rheumatology.

To apply to be a peer reviewer, email your $\mathrm{CV}$ along with a description of your subject areas of interest to eseger@mdedge.com.

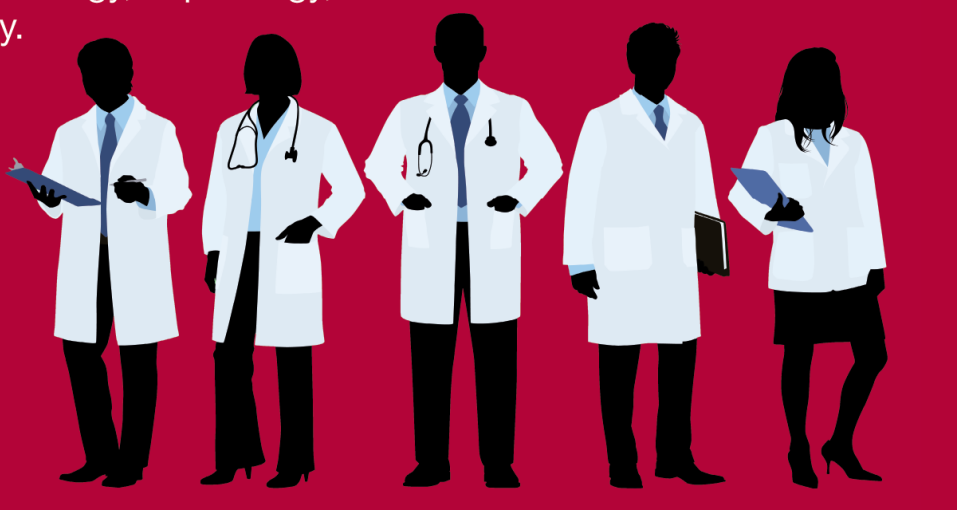




\section{Is Person-Centered Physical Activity-Promoting Intervention for Individuals With CWP More Effective With Digital Support or Telephone Support?}

Juhlin S, Bergenheim A, Gjertsson I, et al. Physical activity with person-centred guidance supported by a digital platform for persons with chronic widespread pain: A randomized controlled trial. J Rehabil Med. 2021;53(4):jrm00175. doi:10.2340/16501977-2796

\section{Study Overview}

Objective. To determine the effectiveness of a personcentered intervention (comprising personalized and cocreated treatment plans to promote physical activity) for individuals with chronic widespread pain when delivered with digital eHealth support compared with standard telephone follow-up.

Design. Single-blinded multicenter randomized controlled trial.

Settings and participants. Participants with chronic widespread pain (CWP) who had participated in a pain management program from 2010-16 at 5 primary health care rehabilitation centers in 5 cities or towns in the western part of Sweden were invited to join the study between March 2018 and April 2019 via letter providing information about the intervention. The letter was followed by a phone call 1-2 weeks later to screen for inclusion and exclusion criteria and interest in participating. Additional participants were invited to participate via a newspaper advertisement in 1 of the 5 cities.

Inclusion criteria were Swedish-speaking persons aged 20-65 years with CWP (defined as having pain in both sides of the body, pain above and below the waist, and axial pain for at least 3 months). Exclusion criteria included having other severe somatic or psychiatric disorders, dominating causes of pain other than CWP, or other severe disease interfering with the ability to be physically active, pregnancy, not having access to a smartphone or a computer, inability to speak or understand Swedish, ongoing physiotherapy treatment, and already exercising regularly. Of 716 people initially assessed for eligibility, 425 completed telephone screening, and 139 were randomized (using block randomization) to either the intervention arm $(n=69)$ or the active control arm $(n=70)$. Due to the nature of the intervention, it was not possible to blind the participants or the physiotherapist to group allocation. All participants provided written informed consent.

The 2 groups underwent the same first individual meeting with a physiotherapist to cocreate a health plan with physical activities, and, if needed, stress management, based on each participant's individual preferences, obstacles, goals, and resources. The difference between the groups was the type of follow-up support. Participants in the intervention group had 1 follow-up meeting with the physiotherapist a week after the initial meeting (to review and adjust the health plan as needed) and thereafter were supported through a digital e-health platform (accessed via the participant's smartphone or computer) during the 6-month follow-up period. Participants were encouraged to access the platform once a week to answer questions regarding their health, and the extent to which they had been able to manage their health plan during the previous week. In addition, the participant and physiotherapist could communicate via the platform as needed. Participants in the active control group had 1 follow-up phone call with the physiotherapist 1 month after the initial meeting (similarly to review and adjust the health plan as needed), and no further contact or support from the physiotherapist during the 6-month follow-up period.

Measures and analysis. The primary outcome measure was pain intensity during the previous week assessed 


\section{Outcomes Research in Review}

with a 0-100 subscale from the Fibromyalgia Impact Questionnaire (FIQ-pain). Secondary outcome measures included overall health status (via FIQ-total with 10 subscales), global fatigue (via FIQ-fatigue subscale), multidimensional fatigue (via Multidimensional Fatigue Inventory, a 20-item questionnaire rated on a 1-5 Likert scale), clinical manifestations of stress (via Stress and Crisis Inventory, a 35-item questionnaire rated on a 0-4 Likert scale), self-efficacy (via General Self-Efficacy Scale, a 10-item questionnaire rated on a 1-4 Likert scale), health-related quality of life (via Short Form 36, specifically the Physical Component Summary composite score), leisure-time physical activity (via Leisure Time Physical Activity Instrument), and physical function (via 1-min chair-stand test). Additional demographic data on age, pain localization, pharmacological treatment, tobacco use, country of birth, level of education, family status, economic status, work status, sick-leave, and disability pension were collected via a questionnaire.

Between-group differences for changes in outcomes from baseline to 6-month follow-up were calculated using the Mann-Whitney $U$ test for continuous data, and Pearson's $\chi^{2}$ or Fisher's exact test for categorical data. Significance level was set at $5 \%$ with no adjustment for multiple comparisons. All analyses were made according to intention-to-treat by originally assigned group; missing cases were not included in the analysis.

Main results. Participants consisted of primarily middle-age, middle income, educated ( $>12$ years of education) females, with $>60 \%$ of participants working at least part-time (between-group differences in baseline data and demographic data not detailed in the article). A total of 29 participants were lost to follow-up. In the intervention group, lost-to-follow up participants were older, performed fewer hours of physical activity, and had lower mental fatigue at baseline, compared with those who were lost to follow-up in the active control group.

In between-group analyses, there were no significant differences in the primary outcome (pain intensity) from baseline to 6-month follow-up. The only significant difference in secondary outcomes was seen in global fatigue the active control group improved significantly compared with the intervention group $(P=.004)$.
In the intervention group, $87 \%$ of participants used the digital platform. Among these users, 35\% contacted the physiotherapist $(75 \%$ of these communications were health- or study-related issues, $25 \%$ were issues with the digital platform), $33 \%$ were contacted by the physiotherapist $(96 \%$ of these communications were about the health plan and physical activity), and 32\% never had any contact with the physiotherapist. There was a significant difference in the primary outcome (pain intensity) from baseline to 6-month follow-up between platform users and non-users $(P=.03$, mean change [SD] 3.8 [19.66] $\mathrm{mm}$ vs -20.5 [6.36] mm, respectively).

Conclusion. No significant differences were found between the groups after 6 months (except for a significant decrease in global fatigue in the active control group compared with the intervention group). Further development of interventions to support persons with CWP to maintain regular physical activity is needed.

\section{Commentary}

Chronic widespread pain is a disorder characterized by diffuse body pain persisting for at least 3 months. ${ }^{1-2}$ It has been associated with lost work productivity, mental ill health, and reduced quality of life. The development of clinically effective and cost-effective pain management strategies for CWP is challenging given the syndrome complexity and heterogenous symptomology. Thus, multimodal, multidisciplinary management is widely advocated, often a combination of education and self-management, with integration of physical, non-pharmacological and pharmacological treatments. ${ }^{1-3}$ Of note, physical exercise and cognitive behavioral therapy are 2 non-pharmacological treatments that hold some promise based on available evidence.

The pervasiveness of technology in nearly all aspects of daily life has corresponded with the development of implementation of a wide range of technology-based interventions for health purposes. ${ }^{4}$ Examples of electronic health or eHealth modalities include internet-based, telephone supported, interactive voice-response, videoconferencing, mobile apps, and virtual reality. While the use of technology in chronic pain management interventions has increased in recent years, the literature is still limited, 
heterogenous, and provides limited evidence on the efficacy of eHealth/digital interventions, let alone which specific modalities are most effective. ${ }^{4-9}$

This study adds to the literature as a randomized controlled trial evaluating the effectiveness of a personcentered intervention for individuals with CWP delivered with digital eHealth support compared with standard telephone follow-up. Results showed no significant difference in the primary outcome of pain intensity and nearly all secondary outcomes between the intervention group (supported by the digital platform) and the active control group (supported by a follow-up phone call). Further, intervention participants who did not use the platform improved significantly more in pain intensity than those who used the platform.

While these results imply that digital support does not contribute to improvements in the outcomes measured, it is important these findings are interpreted with caution given the limitations of the study design as well as limitations with the intervention itself. Importantly, while this study was designed as a randomized controlled trial, the authors indicated that it was not possible to blind the participants or the physiotherapist to group allocation, which may have impacted their behaviors while in the study. In addition, as the authors note, an intervention aimed at increasing physical activity should ideally include an objective measure of activity and this was lacking in this study. The use of an actigraphy device for example would have provided objective, continuous data on movement and could have helped assess an important outcome measure - whether participants reached their physical activity goals or had increased their overall physical activity. In the analysis, there was no adjustment for multiple comparisons or use of imputation methods to handle missing values. Further, it was unclear whether differences in baseline data were evaluated and taken into consideration in between-group analyses. Lastly, results are only attributable to the eHealth mode used in this study (digital web-based with limited mechanisms of behavior change and engagement built-in) and thus should not be generalized to all digital/eHealth interventions persons with CWP.

\section{Applications for Clinical Practice}

While the results of this study failed to demonstrate significant differences between a physical activity-promoting intervention for persons with CWP with digital follow-up vs telephone follow-up, it remains important to consider person-centered principles when offering CWP management support. In this spirit, clinicians should consider a management approach that takes into account the individual's knowledge, resources, and barriers, and also actively involves the patient in treatment planning to enhance the patient's self-efficacy to manage their health. In addition, individual preference for a specific (or combination of) eHealth/digital modality should be considered and used to guide a comprehensive management plan, as well as used as a complementary modality to face-to-face care/support.

\section{-Katrina F. Mateo, PhD, MPH} doi: 10.12788/jcom.0051

\section{References}

1. Bee, P, McBeth, J, MacFarlane, GJ, Lovell K. Managing chronic widespread pain in primary care: a qualitative study of patient perspectives and implications for treatment delivery. BMC Musculoskelet Disord. 2016;17(1):354.

2. Whibley D, Dean LE, Basu N. Management of Widespread Pain and Fibromyalgia. Curr Treatm Opt Rheumatol. 2016;2(4):312-320.

3. Takai Y, Yamamoto-Mitani N, Abe Y, Suzuki M. Literature review of pain management for people with chronic pain. Jpn J Nurs Sci. 2015;12(3):167-183.

4. Slattery BW, Haugh S, O'Connor L, et al. An Evaluation of the Effectiveness of the Modalities Used to Deliver Electronic Health Interventions for Chronic Pain: Systematic Review With Network Meta-Analysis. J Med Internet Res. 2019;21(7):e11086.

5. Heapy AA, Higgins DM, Cervone D, et al. A Systematic Review of Technology-assisted Self-Management Interventions for Chronic Pain. Clin J Pain. 2015;31(6):470-492.

6. Martin CL, Bakker CJ, Breth MS, et al. The efficacy of mobile health interventions used to manage acute or chronic pain: A systematic review. Res Nurs Health. 2021 Feb;44(1):111-128.

7. Bhattarai $P$, Phillips JL. The role of digital health technologies in management of pain in older people: An integrative review. Arch Gerontol and Geriatr. 2017;68:14-24.

8. Bhatia A, Kara J, Janmohamed T, et al. User Engagement and Clinical Impact of the Manage My Pain App in Patients With Chronic Pain: A Real-World, Multi-site Trial. JMIR Mhealth Uhealth. 2021;9(3):e26528.

9. Nevedal DC, Wang C, Oberleitner L, et al. Effects of an individually tailored Web-based chronic pain management program on pain severity, psychological health, and functioning. J Med Internet Res. 2013;15(9):e201. 


\section{Ticagrelor or Clopidogrel in Elective Percutaneous Coronary Intervention}

Silvain J, Lattuca B, Beygui F, et al. Ticagrelor versus clopidogrel in elective percutaneous coronary intervention (ALPHEUS): a randomised, open-label, phase 3b trial. Lancet. 2020;396:1737-44. doi:10.1016/S0140-6736(20)32236-4

\section{Study Overview}

Objective: To assess whether ticagrelor was superior to clopidogrel in reducing periprocedural myocardial necrosis in stable coronary patients undergoing elective percutaneous coronary intervention (PCI).

Design: Multicenter, open-label, and prospective randomized control trial. Setting and participants: A total of 1910 patients with indication for $\mathrm{PCl}$ and at least 1 high risk characteristic were randomized to either ticagrelor or clopidogrel.

Main outcome measures: The primary outcome was the composite of PCl-related type 4a or 4b myocardial infarction or major myocardial injury. The primary safety outcome was major bleeding, evaluated within 48 hours of PCl.

Main results: At 48 hours, the primary outcome was observed in 334 of 941 patients (35\%) in the ticagrelor group and 341 of 942 patients (36\%) in the clopidogrel group (odds ratio [OR], 0.97; 95\% confidence interval [Cl], $0.80-1.17 ; P=.75)$. The primary safety outcome did not differ between groups. Minor bleeding events at 30 days were more frequently observed with ticagrelor (11\%) than clopidogrel (8\%) (1.54; 95\% Cl 1.12-2.11; $P=.007)$.

Conclusion: Among patients undergoing elective PCl, ticagrelor was not superior to clopidogrel in reducing periprocedural myocardial necrosis. Ticagrelor did not cause increase in major bleeding compared to clopidogrel but did increase the rate of minor bleeding at 30 days.

\section{Commentary}

Standard treatment after $\mathrm{PCl}$ includes dual antiplatelet therapy combining adenosine diphosphate (ADP) receptor antagonist and aspirin. The newer generation thienopyridine prasugrel and the reversible direct acting oral antago- nist of the ADP receptor ticagrelor, provides consistent and greater antiplatelet effect compared to clopidogrel, and are superior in reducing ischemic events when compared to clopidogrel in patients presenting with acute coronary syndrome (ACS)., ${ }^{1,2}$ Therefore, current guidelines recommend ticagrelor and prasugrel in preference to clopidogrel in patients presenting with ACS. ${ }^{3,4}$ However, whether these findings of improved outcomes with newer agents compared to clopidogrel extends to patients with stable ischemic heart disease presenting for elective $\mathrm{PCl}$ is unknown.

In this context, Silvain et al investigated this clinical question and compared ticagrelor and clopidogrel by performing a well-designed multicenter randomized control trial in patients presenting with elective $\mathrm{PCl}$. At 48 hours and at 30 days the composite of PCl-related type 4 myocardial infarction or major myocardial injury defined by the third universal definition ${ }^{5}$ was similar between the ticagrelor and clopidogrel groups. Although the incidence of major bleeding was not significantly different between the 2 groups, minor bleeding at 30 days was higher in the ticagrelor group (11\%) than clopidogrel (8\%) $(1.54$; 95\% Cl, 1.12-2.11, $P=.007)$.

The strengths of this current study include the randomized design and the large number of patients enrolled with adequate power to evaluate for superiority of ticagrelor compared to clopidogrel. This was a multicenter trial in Europe with 49 participating centers from France and Czech, and the interventional technique used by the operators reflects contemporary technique with 95\% use of radial or ulnar access.

There are a few important points to consider in this study. First, the primary outcome was biomarker assessed myocardial necrosis and myocardial injury, and the study was not powered to assess the hard outcomes such as death and myocardial infarction. Although there have been previous reports describing the relationship 
between the postprocedural myocardial necrosis with worse outcomes, the definition of myocardial necrosis post- $\mathrm{PCl}$ and its relationship with hard outcomes remains controversial. Second, half of the patients enrolled were on chronic clopidogrel therapy which suggests that patients with inadequate platelet inhibition with clopidogrel may be under-represented in this cohort. Third, this was an open-label study and the knowledge of agent used could have affected the study results. Finally, whether the population included represents a true highrisk population is questionable. Some of the prespecified high-risk features necessary to enter the study was relatively light, such as presence of diabetes mellitus or body mass index $>30 \mathrm{~kg} / \mathrm{m}^{2}$ compared to other criteria such as bifurcation stenting or left main stenting.

Currently, when treating patients with stable ischemic heart disease with higher risk anatomy, some operators may use ticagrelor over clopidogrel by extrapolating the study results from the ACS population. However, the results from the current study do not support the uniform use of ticagrelor in stable patients and suggests that the use of clopidogrel continues to be the standard of care. This is especially relevant considering the cost difference for the 2 agents studied. Whether there is a subgroup that benefits from ticagrelor use, such as patients with unprotected left main stenting or bifurcation stenting with 2 stent strategies, requires further investigation.

\section{Applications for Clinical Practice}

In patients presenting with stable ischemic heart disease undergoing elective $\mathrm{PCl}$, ticagrelor did not lower composite of periprocedural myocardial infarction and myocardial injury at 48 hours. Clopidogrel continues to be a first line treatment after elective $\mathrm{PCl}$.

\section{- Taishi Hirai, MD, and Arun Kumar, MD \\ doi: 10.12788/jcom.0052}

\section{References}

1. Wiviott SD, Braunwald E, McCabe CH, et al. Prasugrel versus clopidogrel in patients with acute coronary syndromes. N Engl $J$ Med. 2007;357(20):2001-15.

2. Wallentin $L$, Becker RC, Budaj $A$, et al. Ticagrelor versus Clopidogrel in Patients with Acute Coronary Syndromes. $N$ Engl $J$ Med. 2009;361(11):1045-57.

3. Ibanez B, James S, Agewall S, et al. 2017 ESC Guidelines for the management of acute myocardial infarction in patients presenting with ST-segment elevation: The Task Force for the management of acute myocardial infarction in patients presenting with ST-segment elevation of the European Society of Cardiology (ESC). Eur Heart J. 2018;39(2):119-177.

4. Levine GN, Bates ER, Bittl JA, et al. 2016 ACC/AHA guideline focused update on duration of dual antiplatelet therapy in patients with coronary artery disease: A report of the American College of Cardiology/American Heart Association Task Force on Clinical Practice Guidelines. J Thorac Cardiovasc Surg. 2016;152(5):12432-1275.

5. Thygesen K, Alpert JS, Jaffe AS, et al. Third universal definition of myocardial infarction. J Am Coll Cardiol. 2012;60(16):1581-98. 